\section{Neuzulassung bei KHK}

Der orale Gerinnungshemmer Rivaroxaban (Xarelto ${ }^{\circledR}$ ) ist im Mai 2013 von der Europäischen Kommission zur Prävention atherothrombotischer Ereignisse (kardiovaskuläre Sterblichkeit, Myokardinfarkt oder Schlaganfall) nach akutem Koronarsyndrom bei Patienten mit erhöhten kardialen Biomarkern zugelassen worden (Dosierung: zweimal täglich 2,5 mg). Die zulassungsrelevanten Daten stammen aus der Studie ATLAS ACS 2-TIMI 51 mit mehr als 15.500 Patienten, in der Rivaroxaban kardiovaskuläre Ereignisse additiv zur Standardtherapie mit Plättchenhemmern stärker reduzierte als die Standardtherapie allein.

Ärzte Zeitung, 02.07.2013

\section{Infos im Internet}

Aktuelle Informationen zu kardiologischen Themen bietet das Onlineportal www.kardiologie.org, das von der DGK mit Springer Medizin als Partner betrieben wird. Dort finden Sie aktuelle Berichte zu Studien und Kongressen, Inhalte aus Springer-Fachmedien sowie einen kardiologischen Expertenrat.

Leitlinien, Kommentare, Positions- und Konsensuspapiere zu kardiologischen Indikationen sind auf der Leitlinien-Website der DGK unter http://leitlinien.dgk.org/ zu finden.

\section{Daten und Fakten}

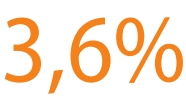

betrug im Jahr 2011 der Rückgang von diagnostischen und therapeutischen Linksherzkatheteruntersuchungen in Deutschland im Vergleich zum Vorjahr. In all den Jahren zuvor hatten die im jährlichen „Herzbericht" präsentierten Leistungszahlen stets eine Zunahme dokumentiert. Experten sprechen bereits von einer "Trendwende“. Die Zahlen zeigen auch, dass die Patienten immer älter werden. Über 50\% der Katheter-Eingriffe werden heute bei über 70-Jährigen durchgeführt.

Was ist die richtige Option?

\title{
Bypass-Op. versus PCI
}

Orientierung in der Grenzzone von interventioneller Kardiologie und Herzchirurgie verschaffen die Ergebnisse der SYNTAX-Studie.

In der SYNTAX-Studie ging es um nichts weniger als die Frage, welche dieser beiden Fachgruppen mit ihrer jeweiligen Methode der Revaskularisation für Patienten mit fortgeschrittener KHK die bestmögliche Behandlung $\mathrm{zu}$ bieten hat. Im Februar 2013 sind in "The Lancet" die finalen 5-Jahres-Ergebnisse veröffentlicht worden.

Vorteil der Op. bei komplexen Fällen In die Studie sind 1800 Patienten mit koronarer Dreigefäßerkrankung und/oder Stenose des linken Hauptstamms aufgenommen worden. Von den Teilnehmern sind 897 einer Bypass-Op. und 903 einer PCI als Revaskularisationsverfahren zugeteilt worden. Die Bilanz nach fünf Jahren macht deutlich, dass der Vorteil der
Herzchirurgie umso deutlicher ausfiel, je komplexer der Koronarbefund - gemessen am SYNTAX-Score - war. Bei Patienten mit weniger ausgedehntem Koronarbefall erwies sich dagegen die interventionelle Behandlung als mindestens gleichwertig. Bei den Endpunkten Tod oder Schlaganfall gab es nach fünf Jahren keinen signifikanten Unterschied zwischen chirurgischer und interventioneller Revaskularisation (Mortalität: 11,4\% vs. 13,7\%). Die Bypass-Op. offenbarte dann signifikante Vorteile, wenn als Vergleichsmaßstab die Häufigkeit von Herzinfarkten (3,8\% vs. 9,7\%) und von wiederholten Revaskularisationen $(13,7 \%$ vs. $25,9 \%)$ herangezogen wurde. Eindeutig Boden gut gemacht hat die interventionelle Stent-Therapie mit der SYNTAX-Studie in der Behandlung von Hauptstammstenosen, die bisher als Domäne der Herzchirurgie galt.

(ob)

Ärzte Zeitung, 02.07. 2013

\section{Studie abgebrochen}

\section{HDL-Erhöhung ohne sichtbaren Erfolg}

Das „böse“ LDL-Cholesterin muss runter, das "gute" HDL-Cholesterin muss rauf - mittlerweile ein fraglicher Grundsatz.

Ob sich aber nach dieser simplen Faustregel Herzinfarkte verhindern lassen, ist - zumindest was das HDL-Cholesterin betrifft - mittlerweile sehr fraglich. Ausgehend von der Beobachtung in epidemiologischen Studien, dass hohe HDL-Spiegel mit einem niedrigen kardiovaskulären $\mathrm{Ri}$ siko assoziiert sind, schien die medikamentöse HDL-Erhöhung ein guter Ansatzpunkt zur Prävention kardiovaskulärer Erkrankungen zu sein. Die Bestätigung lässt aber noch auf sich warten. Eine große Endpunktstudie mit dem CETP-Modulator Dalcetrapib, der das HDL-Cholesterin moderat um etwa $30 \%$ erhöht, ohne das LDL-Cholesterin wesentlich zu verändern, ist im Mai 2012 wegen Wirkungslosigkeit vorzeitig beendet worden.

\section{Studienziele nicht erreicht}

Die nächste Enttäuschung ließ nicht lange auf sich warten. Ende 2012 wurde bekannt, dass auch die große HPS2-THRIVE-Studie ihr Ziel nicht erreicht hat. Trotz moderater HDL-Erhöhung und LDL-Senkung mit einem Präparat, das Nikotinsäure (Niacin) in Kombination mit dem Anti-Flush-Wirkstoff Laropiprant enthielt, war es nicht gelungen, additiv zu Statinen die Inzidenz vaskulärer Ereignisse weiter zu senken.

(ob)

Ärzte Zeitung, 02.07.2013 